[Agr. Biol. Chem., Vol. 29, No. 7, p. 662 664, 1965]

\title{
Blätteralkohol (XIV)
}

\author{
Synthese des 2-Propyl-5-äthyl-benzylalkohols \\ von Akikazu Hatanaka, Tadahiko Kajiwara und Minoru Ohno
}

Eingegangen am 29 Januar 1965

\begin{abstract}
2-Propyl-5-ethyl-benzylalcohol (IX) was synthesized by an unequivocal route from propylbenzene, thereby establishing the previous deduction tentatively assigned to the leaf alcohol reaction product.1) This benzyl alcohol surmises one of a lemon-like flavor characteristic of manufactured black tea and an attempted search for this compound in the essential oil obtained by steamdistillation of manufactured black tea was made, but its existence has not so far been confirmed with a neutral fraction examined.
\end{abstract}

In den letzten Mitteilung ${ }^{1)}$ erhielten wir durch Erhitzen des Blätteralkohols mit $\mathrm{Na}$ ein nach Orangen riechende Alkohol. Damals wurde sein Struktur durch sein Oxydation, die Synthese der analogen Substanzen und mit UV- sowie IR-Spektren als 2-Propy-5äthyl-benzylalkohol aufgeschlossen. In dieser Mitteilung um die Struktur synthetisch zu erklären, versuchten wir hier sein Alkohol aus den armatischen Verbindung darzustellen. Der Syntheseweg ist wie folgendes Schema. Das 3,5-Dinitrobenzoat des erhaltenen Alkohols zeigte keine Schmelzpunktserniedrigung mit dem des aus dem Blätteralkohol erhaltenen Alkohols.

Bei dieser Synthese wurden die folgenden Tatsache aufgefunden.

1) Die Substanz (II) wurde gaschromatographisch als Substanz, die iso-Propyl-benzol nicht enthielt, nachgewiesen.

2) Das Semicarbazon der Substanz (III) schmolz bei $186.5 \sim 176^{\circ} \mathrm{C} \quad\left(\right.$ Lit. $^{2)}$ Schmp. $180 \sim 180.5^{\circ} \mathrm{C}$ ). Die aus dem Semicarbazon durch Hydrolyse erhaltene Substanz ergab sich im Gaschromatogramm drei Peaks. Davon wurde durch prazise Fraktionierung nur 4Propylacetophenon (III) erhalten.

1) Minoru Ohno, Akikazu Hatanaka und Yuzo Inouye; Agr. Biol. Chem., 26, 460 (1962).

2) O. Widman; Ber., 21, 2224 (1888).
3) Die Reduktion des 3-Amino-4-Propylacetophenon (V) zu 3-Amino-4-Propyl-äthylbenzol (VI) durch WOLFF-KISHNER-Reduktion in fast quantitativ verführt.

4) Die Diazotierung des (V) lief sehr schwerig. Es konnte auf die sterische Hinderung der $n$-Propyl-Gruppe zurückgeführt werden.

5) Die GRIGNARD-Verbindung des (VII) wurde mit grösser Schwierigkeit hergestellt; Erst nach dem 6 stdn. Rückfluss entstand die gewunschte Verbindung.

Prüfung nach Vorkommen des 2-Propyl-5äthyl-benzylalkonols im Schwarzen Tee.

Im Gesichtpunkt aus Ähnlichkeit des Riechens dieses 2-Propyl-5-äthyl-benzylalkohols mit dem nach Orangen Riechen wurde es möglich vermutet, dass 2-Propyl-5-äthylbenzylalkohol in dem Schwarzen Tee enthalten ist. Wir haben ersucht aus dem Schwarzen Tee dieselbe Substanz nachzuweisen. $684 \mathrm{Kg}$ Schwarzer Tee wurde mit Wasserdampf untergebracht, und ätherisches Öl wurde erhalten. Durch die Fraktionierung des davon erhaltenen neutralen Teils wurde die der Siedepunkt des 2-Propyl-5-äthyl-benzylalkohols entsprechende Fraktion getrennt. Sie stimmt nicht mit 2-Propyl-5-äthyl-benzylalkohol in IR-Spektren und in Gaschromatogramm überein. Nach Behandlung mit Phtalsäureanhydrid wurde kein primäre Alkohol erhalten. 
Schema<smiles>ClCc1ccccc1</smiles>

(I)<smiles>CCCc1ccc(C(C)=O)cc1[N+](=O)[O-]</smiles>

(IV)<smiles>CCc1ccc(CC)c(Br)c1</smiles>

(VII)
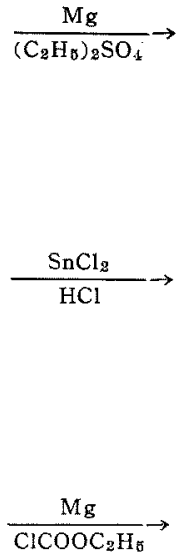

(VIII)

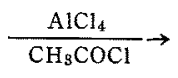

(II)<smiles>CCCc1ccc(C(C)=O)cc1N</smiles>

(V)<smiles>CCCCc1ccc(CC)cc1C(=O)OCC</smiles>

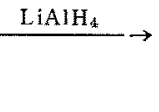

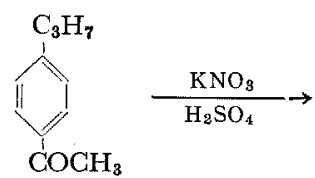

(III)<smiles>CCCc1ccc(CC)cc1N</smiles>

(VI)<smiles>CCc1ccc(CC)c(CO)c1</smiles>

(IX)

\section{BESCHREIBUNG DER VERSUCHE}

\section{I) Synthese des 2-Propyl-5-äthyl-benzylalkohols}

n-Propyl-benzol (II): Unter völligem Feuchtigkeitsausschluss und dem Rühren gab man zu $48.6 \mathrm{~g}$ feinen Mg-Spänen im $200 \mathrm{ml}$ absol. Äther in gelindem Sieden tropfenweise $253 \mathrm{~g}$ Benzylchlorid (I) in $1000 \mathrm{ml}$ absol. Äther. Nach Zugabe wurde das Gemisch noch 1 Stde. unter Rückfluss auf dem wasserbad erhitzt. Unter Eiskühlung wurden $616 \mathrm{~g}$ frisch destillierter Diäthylsulfat im Gemisch tropfenweise zufügt. Nach 15 min. Rückfluss wurde das Reaktionsprodukt in $20 \% \mathrm{HCl} / 1 \mathrm{~kg}$ Eisstück eingegossen. Die wässr.-Phase wurde mit $4 \times$ je $300 \mathrm{ml}$ Äther ausgeschüttelt und die davon erhaltene Ätherlösung wurde mit der ätherische Phase versetzt. Nach Abdestillieren des Äthers wurde es mit $10 \% \mathrm{NaOH}-\mathrm{Lösung}$ in $50 \% \mathrm{EtOH}$ versetzt und dann durch Rückfluss bei 1 Stde. wurde die überschüsssige Diäthylsulfat abgespaltet. Dazu wurde Wasser zugeben und es wurde mit Äther geschüttelt und nach dem Trocknen mit $\mathrm{KOH}$ wurde rohes nPropyl-benzol bei $154 \sim 9^{\circ} \mathrm{C}$ erhalten, $n_{\mathrm{D}}^{20} 1.4931$, Ausb. $180 \mathrm{~g}(75 \%$ d. Th.).

4-Propyl-acetophenon (III): Unter dem heftigen Rühren wurde $60 \mathrm{~g} n$-Propyl-benzol (II) mit $66 \mathrm{~g}$ Aluminium chlorid pulver versetzt. Unter Eiskühlung bei $120^{\circ} \mathrm{C}$ (inner Temperatur) wurden $78 \mathrm{~g}$ Acetylchlorid zudem tropfenweise gegeban. Im Schliessen der HCl-
Entwicklung wurde es zu Eis-Wasser eingegossen. Nach dem Auswaschen des Reaktionsprodukts mit Wasser und dem Ausschütteln mit Äther wurde die Ätherlösung mit $\mathrm{CaCl}_{2}$ getrocknet. Nach dem Abdestillieren des Äthers siedete (III) bei $95^{\circ} \mathrm{C} / 2 \mathrm{~mm}, n_{\mathrm{D}}^{20} 1.5215$, Ausb. $58 \mathrm{~g}$ (71.4\% d. Th.).

Semicarbazon: Aus Äthylalkohol in silberig glänzenden, weissen Blätterchen. Schmp,; $84 \sim 5^{\circ} \mathrm{C}$ (Lit.: $\left.180 \sim 180.5^{\circ} \mathrm{C}\right)$, aus Benzol/Hexan, Schmp. 186.5 $187^{\circ} \mathrm{C}$.

Gaschromatogramm der aus dem Semicarbazon durch Hydrolyse erhaltene Substanz:

Es hat drei Peaks und die Retentionszeit ist je 5.1, 7.7 und $12.3 \mathrm{~min}$. (Die Versuchsbedingungen waren: Trägergas; Helinm, mit einer Strömungsgeschwindigkeit von etwa $60 \mathrm{ml} / \mathrm{min}$. bei $175^{\circ} \mathrm{C}$, Kolonne: PEG-6000; $0.6 \times 0.6 \mathrm{~m}$.).

Durch Fraktioniersrohr (50 Kolonne) siedende 4Propyl-acetophenon, das im Gaschromatogamm mit $12.3 \mathrm{~min}$. Retentionszeit stimmte überein, bei $96 \sim$ $8^{\circ} \mathrm{C} / 4 \mathrm{~mm}$.

3-Nitro-4-propyl-acetophenon (IV): Diese Verbindung (IV) bildet sich in guter Ausbeute, wenn $13.4 \mathrm{~g}$ 4-Propyl-acetophenon (III) unter kräftigem Umrühren und Kühren mit Eis-Kochsalzmischung in $\mathrm{KNO}_{3}$-konz. $\mathrm{H}_{2} \mathrm{SO}_{4}$-Lösung $\quad\left(10.3 \mathrm{~g} \quad \mathrm{KNO}_{8} / 100 \mathrm{~g}\right.$ $\mathrm{H}_{2} \mathrm{SO}_{4}$ ) getropft werden. Das Gemisch wurde bei 
$5^{\circ} \mathrm{C}$ noch 1 Stde. gerührt. Das Reaktionsprodukt wurde auf Eis gegossen, wobei sich keine Spur Öl abschied. Nach Abdestillieren des Äthers siedete die äther. Auszügen bei $122 \sim 4^{\circ} \mathrm{C} / 0.05 \mathrm{~mm}, n_{\mathrm{D}}^{18} 1.5400$, Schmp. $57 \sim 60^{\circ} \mathrm{C}$, Ausb. $17 \mathrm{~g}(94.9 \%$ d. Th.).

3-Amino-4-propyl-acetophenon (V): Unter Kührung mit Wasser wurden $52.5 \mathrm{~g}$ Zinnchlorür in $20 \mathrm{ml}$ konz. $\mathrm{HCl} z$ dem $15.5 \mathrm{~g}$ 3-Nitro-4-propyl-acetophenon in $50 \mathrm{ml} \mathrm{MeOH}$ gegossen. Nach noch is Stdn. Umrühren und Erwärmen wurde $\mathrm{MeOH}$ abdestillt und dann Überschuss von $40 \%$-iger Natronlauge wurde in diesem Reaktionsgemisch langsam gegossen. Die Base wurde mit Äther ausgeschüttelt und nach Abdestillieren des Äthers ging ( $V$ ) bei $122 \sim 4^{\circ} \mathrm{C} / 0.25 \mathrm{~mm}$ über, $n_{\mathrm{D}}^{13}$ 1.5792, Schmp. $36^{\circ} \mathrm{C}$, Ausb. $11 \mathrm{~g}(82.6 \%$ d. Th.).

3-A mino-4-propyl-âthylbenzol (VI): $3.5 \mathrm{~g}$ 3-Amino4-propyl-acetophenon (V) wurden mit $2 \mathrm{~g} 90 \%$ Hydrazinhydrat $2 \mathrm{Stdn}$. auf $140^{\circ} \mathrm{C}$ gekocht. Das Gemisch wurde mit Wasser ausgekührt und dann wurde $27 \mathrm{~g}$ Kaliumhydroxyd-pulver in dem zugeben und das Gemisch wurde auf $200^{\circ} \mathrm{C} 6 \mathrm{Stdn}$. erhitzt. Das Reaktionsprodukt wurde in Wasser eingegossen und mit Äther ausgeichüttelt. Nach Abdestillieren des Äthers wurde (VI) bei $71^{\circ} \mathrm{C} / 4 \mathrm{~mm}, n_{\mathrm{D}}^{15}$ 1.5379, Ausb. $3.23 \mathrm{~g}$ (99.08\% d. Th.).

3-Acetoamino-4-propyl-äthylbenzol: Aus Äthylalkohol, Schmp. $138^{\circ} \mathrm{C}, \mathrm{C}_{13} \mathrm{H}_{19} \mathrm{ON}$ (205.29):

Ber. C, 76.05; H, 9.33; N, 6.82

Gef. C, 76.12; H, 9.47; N, 6.99

3-Brom-4-Propyl-äthylbenzol(VII): Unter Kühlung mit Eis-Wasser wurden $80 \mathrm{ml} 44 \%$ Bromwasserstoffsäure in $12.25 \mathrm{~g} \mathrm{3-Amino-4-propyl-äthylbenzol} \mathrm{tropfen-}$ weise zugeben. (VI)-HBr wurde mit Eis-Kochsalzmiscung bei $-10^{\circ} \mathrm{C}$ ausgekührt und dann wurde es mit $5.8 \mathrm{~g} \mathrm{NaNO}_{2}$ diazotiriert. Wobei kam diese Reaktionstemperatur über $13^{\circ} \mathrm{C}$ nicht. Unter Kühlung wurden $0.5 \mathrm{~g}$ Kupfer-pulver in der gerührt. Nachdem noch $\frac{1}{2}$ Stde, Diazoverbindung zugeben und bei $10^{\circ} \mathrm{C}$ noch $\frac{1}{2} \mathrm{Stde}$, bei $60^{\circ} \mathrm{C}$ wurdedas $\mathrm{Gemisch}$ auf Wasserbad erhitzt. Das nach dem Wasserdampfdestillation des Reaktionsprodukts erhaltene Destillat wurde mit überschüssigem KOH versetzt. Nach Waschen mit verdünnte Schwefelsäure wurde es mit Äther ausgeschuttelt. Nach dem Abdestillieren des Äthers wurde (VII) erhalten, Sdp. $138 \sim 9^{\circ} \mathrm{C} / 14 \mathrm{~mm}, n_{\mathrm{D}}^{18} 1.5235$, Ausb. $7.3 \mathrm{~g}(43 \%$ d. Th.). Sein IR-Spektrum zeigte die Absorption der geringen $\mathrm{OH}-\mathrm{Gruppe}$.

2-Propyl-5-äthyl-benzoesăureäthylester (VIII): $5.22 \mathrm{~g} \mathrm{3-Brom-4-propyl-äthylbenzol} \mathrm{in} 20 \mathrm{ml}$ absol. Ather wurden in $700 \mathrm{mg}$ feinen Mg-Spänen in $15 \mathrm{ml}$ absol. Äther zugeben. Unter Rückfluss 6 Stdn. wurde das Gemisch auf Wasserbad erwärmt. Die Präparierung der GRIGNARD-Verbindung verführte mit grösser Schwierigkeit. $\mathrm{Zu}$ dieser GRIGNARDVerbindung wurden $2.7 \mathrm{~g}$ Chlorcarbonsäureäthylester tropfenweise zugeben. Nach $2 \mathrm{Stdn}$. Rückfluss wurde das Reaktionsgemisch mit 10\% HCl behandelt. Nach dem äther. Ausschütteln und dem Abdestillieren des Athers ging (VIII) bei $94 \sim 5^{\circ} \mathrm{C} / 4 \mathrm{~mm}$ über, $n_{\mathrm{D}}^{12} 1.5020$, Ausb. $2.1 \mathrm{~g}$, (40\% d. Th.).

2-Propy l-5-äthyl-benzylalkohol (IX): $2 . \lg$ (VIII) in $10 \mathrm{ml}$ absol. Äther wurden in den $360 \mathrm{mg} \mathrm{LiAlH}_{4}$ in $20 \mathrm{ml}$ absol. Äther langsam unter Rühren zutropft (die $\mathrm{LiAlH}_{4}$-Suspension soll zumindest $30 \mathrm{Min}$, vor der ersten Zugabe von äther. Esterlösug bereitet sein). Die Zugabe erfolgte in dem Masse, dass die Mischung stets in gelindem Sieden blieb; zuletzt wurde das etwa zäh gewordene Reaktionsgemisch schwach erwärmt Durch Zugabe von $10 \% \mathrm{HCl}$ wurde es zergesetzt. Nach dem äther. Ausschütteln und Abdestillieren des Äthers wurde 2-Propyl-5-äthyl-benzylalkohol bei $136 \sim 9^{\circ} \mathrm{C} / 12 \mathrm{~mm}$ erhalten, $n_{\mathrm{D}}^{20} 1.5210$, Ausb. $1.2 \mathrm{~g}$ (70.5\% d. Th.).

3,5-Dinitrobenzoat des (IX): Aus Äthylalkohol Schmp. $78^{\circ} \mathrm{C}$. Sein 3,5 -Dinitrobenzoat zeigte keine Schmelzpunktserniederung mit dem des aus dem Blätteralkohol erhaltenen Alkohols.

Prăparieren der mit der Siedepunkt des 2. Propy-5-äthyl-benzylalkohols entsprechenden Alkoholfraktion aus schwarzem Tee: $7 \mathrm{~kg}$ Frischer schwarzem Tee wurden mit $6 \mathbf{L}$ Wasser versetzt und das Gemisch wurde geknetet. Nach Wasserdampfdestillation wurden etwa $50 \mathrm{~L}(3.5 \mathrm{~L} \times 14)$ Destillat erhalten und mit $\mathrm{NaCl}$ gesättigt. Je $3.5 \mathrm{~L}$ Destillat

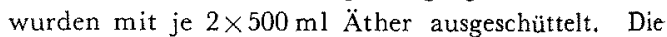
durch die gleiche Behandlung aus $684.8 \mathrm{~kg}$ erhaltene äther. Lösung wurde langsam Äther abdestilliert. Die davon erhaltene etwa $200 \mathrm{~g}$ äther. Auszugen wurden mit gesät. $\mathrm{NaHCO}_{3}$-Lösung, $5 \% \mathrm{NaOH}$-Lösung und $3 \% \mathrm{HCl}$ behandelt und $118.71 \mathrm{~g}$ neutraler Teil wurden gewonnen. Durch Destillieren des neutralen Teils wurden $4.2 \mathrm{~g}$ die Fraktion bei $124 \sim 150^{\circ} \mathrm{C} / 12 \mathrm{~mm}$ Propyl-5-äthyl-benzylalkohol: Sdp. $\quad 138 \sim 9^{\circ} \mathrm{C} / 12 \mathrm{~mm}$ ) erhalten, $n_{\mathrm{D}}^{20}$ 1.4940. Sein IR-Sektrum zeigte fast nicht die Absorption der OH-Gruppe. Die Fraktion zeigte nicht in Gaschromatogramm die mit 2-Propyl-5-athylbenzylalkohol entsprechende Retentionszeit. $4.2 \mathrm{~g}$ die Fraktion wurden mit $4.0 \mathrm{~g}$ Phtalsäureanhydrid in absol. Benzol behandelt. Aber die primäre Alkohols wurde nicht gewonnen. 